\title{
Low-cost methodology for skin strain measurement of a flexed biological limb
}

\author{
Bevin Lin, Kevin M. Moerman, Connor G. McMahan, Kenneth A. Pasch, and Hugh M. Herr*
}

\begin{abstract}
Objective: The purpose of this manuscript is to compute skin strain data from a flexed biological limb, using portable, inexpensive, and easily-available resources. Methods: We apply and evaluate this approach on a person with bi-lateral transtibial amputations, imaging left and right residual limbs in extended and flexed knee postures. We map 3D deformations to a flexed biological limb using freeware and a simple point-and-shoot camera. Mean principal strain, maximum shear strain, as well as lines of maximum, minimum, and non-extension are computed from 3D digital models to inform directional mappings of the strain field for an unloaded residual limb. Results: Peak tensile strains are $\sim 0.3$ on the anterior surface of the knee in the proximal region of the patella, whereas peak compressive strains are $\sim \mathbf{- 0 . 5}$ on the posterior surface of the knee. Peak maximum shear strains are $\sim 0.3$ on the posterior surface of the knee. The accuracy and precision of this methodology are assessed for a ground truth model. The mean point location distance is found to be $0.08 \mathrm{~cm}$, and the overall standard deviation for point location difference vectors is $0.05 \mathrm{~cm}$. Conclusion: This low-cost and mobile methodology may prove critical for applications such as the prosthetic socket interface where whole-limb skin strain data are required from patients in the field outside of traditional, largescale clinical centers. Significance: Such data may inform the design of wearable technologies that directly interface with human skin.
\end{abstract}

Index Terms-skin strain, lines of non-extension, wearable technology.

\section{INTRODUCTION}

$\mathrm{W}$ hole-limb skin strain analysis may prove critical to the design of wearable devices that mechanically interface with the human body. In this manuscript, skin strain of a flexed biological limb is measured using highly portable and inexpensive resources, conducive for collecting data in the field, unconfined to a laboratory setting. Such strain data, and biomechanical models derived from these data, may provide insight into the general design of broad applications including apparel, shoes, prostheses, orthoses, body exoskeletons, spacesuits, and other wearable devices where safe and comfortable mechanical loading is sought between the synthetic product and the body [1], [2]. Measuring skin strain is a challenge given its heterogeneous mechanical properties. The multi-layered composition of fibrous, gelatinous, and glandular tissues, as well as nerves and blood vessels, cause skin to behave as a

This work was supported by the Robert Wood Johnson Foundation.

B. Lin is with the Center for Extreme Bionics, MIT, Cambridge, MA 02139 (e-mail: bevinlin@media.mit.edu).

K.M. Moerman is with the Center for Extreme Bionics, MIT, Cambridge, MA 02139 (e-mail: kmoerman@media.mit.edu). fluid-solid mixture [3] with non-linear elastic [4] and viscoelastic properties [5]. Furthermore, skin biomechanical properties are subject to variation based on dermal thickness, age, sun exposure, tissue composition, and location on the body [6]. Despite being the largest organ of the body, these characteristics make skin very challenging to study, due in part to intricate hardware, software, and environmental requirements [7].

Digital image correlation (DIC) is a powerful technique for skin strain assessment based on ease of application, high resolution, and available turn-key integrated software. Commercial DIC systems have been used successfully to analyze 3D surface deformation [8], and have been combined with finite element modeling to study skin mechanical properties in-vivo [9]. DIC skin strain investigations across a joint collect surface data between flexed and extended states, followed by a ray tracing technique to reconstruct 3D displacements [10]. Although semi-portable, DIC systems can be costly. Furthermore, the technique has largely been confined to analysis of near-planar imaging surfaces. As a solution to this limitation, motion capture systems have successfully characterized whole-limb in vivo strain of a flexed biological limb [2], [11], [12][13][14].

TABLE I

COMPARISON OF SKIN STRAIN ANALYSIS METHODS

\begin{tabular}{|c|c|c|c|}
\hline & $\mathrm{DIC}^{\mathrm{a}}$ & Motion capture ${ }^{b}$ & Point and shoot \\
\hline System cost & $>\$ 150 \mathrm{~K}$ & $>\$ 150 \mathrm{~K}$ & $<\$ 300$ \\
\hline $\begin{array}{c}\text { Data } \\
\text { acquisition } \\
\text { environment }\end{array}$ & $\begin{array}{l}\text { Laboratory, } \\
\text { semi-portable }\end{array}$ & Laboratory & Portable \\
\hline $\begin{array}{c}\text { Vendor } \\
\text { purchase, set-up }\end{array}$ & 4-6 weeks & 4-6 weeks & $\sim 1$ hour \\
\hline Resolution & $\begin{array}{c}\text { Skin pattern } \\
\text { density }\end{array}$ & $\begin{array}{c}\text { Reflective } \\
\text { marker limited }\end{array}$ & $\begin{array}{c}\text { Skin pattern } \\
\text { density }\end{array}$ \\
\hline Dimensionality & Planar & $3 \mathrm{D}$ & $3 \mathrm{D}$ \\
\hline
\end{tabular}

a integrated turn-key hardware and software system for data acquisition and analysis

${ }^{\mathrm{b}}$ includes gait lab and data acquisition hardware and software

Given whole-limb, 3D capability of motion capture techniques, lines of non-extension (LONE), lines where skin does not stretch or compress [15], can be estimated. While motion capture systems provide important information, they are

C.G. McMahan is with the Mechanical Engineering Department, MIT, Cambridge, MA 02139 (e-mail: cmcmahan@mit.edu).

K.A. Pasch is with the Center for Extreme Bionics, MIT, Cambridge, MA 02139 (e-mail: kenpasch@media.mit.edu).

*H.M. Herr is with the Center for Extreme Bionics, MIT, Cambridge, MA 02139 (e-mail: hherr@media.mit.edu). 
costly, confined to a laboratory environment, and resolutionlimited to the size and placement of 3D position trackers. Additionally, data collection is often impeded due to loss of tracking visibility where the detectors cannot locate the reflective markers. Table I compares key components of DIC, motion capture, and the low-cost point-and-shoot and highly mobile method described in this work.

The objective of this study is to present and assess a low-cost methodology $(<\$ 300)$ for quantifying whole-limb skin strain across a joint. Skin strain is calculated using direct skin markings, a simple point-and-shoot camera, and publicly available software to analyze static 3D human body postures. The proposed method acquires whole-limb strain data comparable to DIC and motion capture systems but without their inhibitory cost and lack of portability. As such data may inform the design of future prosthetic socket interfaces, the method is evaluated by analyzing skin strain for a flexed knee joint in a human participant with bi-lateral transtibial amputations.

\section{MATERIALS AND METHODS}

\section{A. Human Participants}

The Committee On the Use of Humans as Experimental Subjects at the Massachusetts Institute of Technology (MIT) approved this research investigation. One healthy adult male with bilateral transtibial amputations consented and enrolled in this pilot study. Data are collected from the left residual limb (n1), and the right residual limb (n2) to comprise two independent data sets of both extended and flexed postures.

\section{B. The Skin Strain Measurement Process}

Two static knee joint postures, extended $\left(\sim 0^{\circ}\right.$ flexion, or full extension) and flexed $\left(\sim 60^{\circ}\right.$ flexion) are considered for this study. Skin strain is measured between these two postures for both the left and right residual limb. Each step of the skin strain quantification process is shown in the schematic overview of Fig. 1. Steps 1-4 are completed for each residual limb (left and right leg) and each knee posture (extended and flexed). Step 5 corresponds the extended and flexed postures, necessary for strain computation in Step 6. These steps are detailed below.

\section{Step (1): Skin Marker Pattern Application}

A dotted marker pattern is manually applied across the residual limb surface (Fig. 2) with non-toxic black body ink (Milani Cosmetics, Los Angeles, CA). The black ink was determined to be optimal based on a color palette analysis across RGB and black body paints. Dark ink is highly contrasting for light skin-tone. Light ink is highly contrasting for dark skin-tone. Markers are $3 \mathrm{~mm}$ diameter circles, positioned approximately $2 \mathrm{~cm}$ apart (a distance less than the 4 $\mathrm{cm}$ two-point discrimination of human skin on the medial surface of the lower leg [16]). The marker pattern is applied such that the markers closely represent the vertices of an

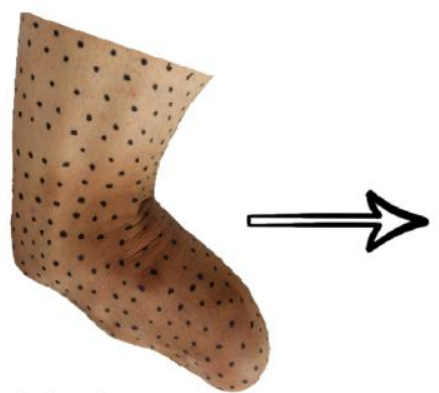

1. Marker pattern application

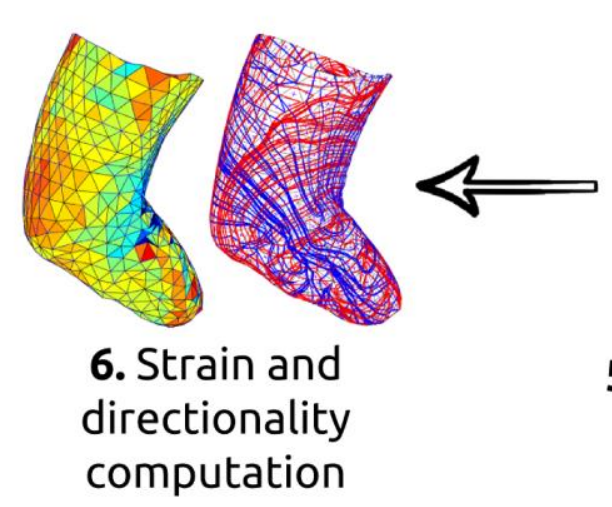

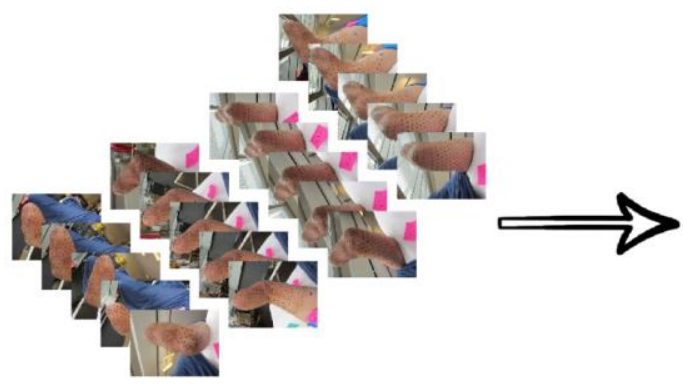

2. Image acquisition

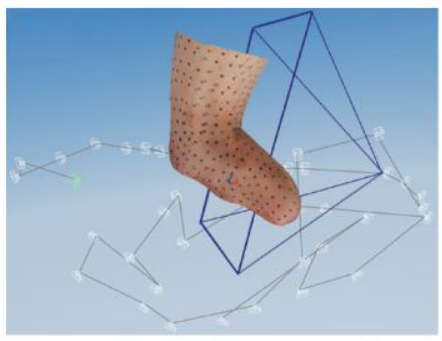

3. Textured 3D surface model construction

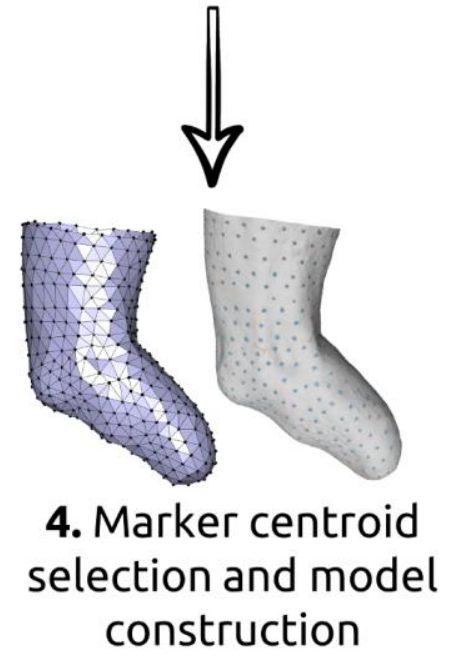

Fig. 1. Schematic overview of the strain measurement procedure. Step 1: a marker pattern is applied to the skin surface. Step 2: photographs are acquired $360^{\circ}$ around the residual limb. Step 3: images are processed to create a 3D textured surface model. Step 4: the marker centroids are identified across the limb to create a triangulated marker centroid surface model. Step 5: identical markers in the extended and flexed state are corresponded. Step 6: the corresponded marker models are used for skin strain and strain directionality (e.g. LONE) computation. 
equilateral triangular pattern. Such a pattern aids in marker surface model construction and is necessary for point correspondence and strain computation (detailed below in Steps 4-6). During marker application, the anatomical location and spacing need not be precise. However, the marker density (number of markers per $\mathrm{cm}^{2}$ ) is important, as this defines the resolution of the resulting skin strain field. The chosen marker size and spacing maximizes point density while keeping computational times to within 1-3 hours. Given our intended application of strain-informed prosthetic interface design, we mark an area encompassing the typical region of the leg in contact with a prosthetic liner (from the distal end to $\sim 15-20 \mathrm{~cm}$ above the knee). Initiating at the most proximal row, a tape measure is used to manually place each marker $\sim 2 \mathrm{~cm}$ apart. These spaced markers also serve as scaling dimensions for the 3D digital model. Natural skin tone variations, moles, and marker diameter/shape variations provide image texture and non-uniformity, necessary for pattern recognition during model reconstruction. Fig. 2 shows the final inked marker pattern for a transtibial amputated limb in the extended and flexed posture. The process to mark the skin takes approximately 30-45 minutes.
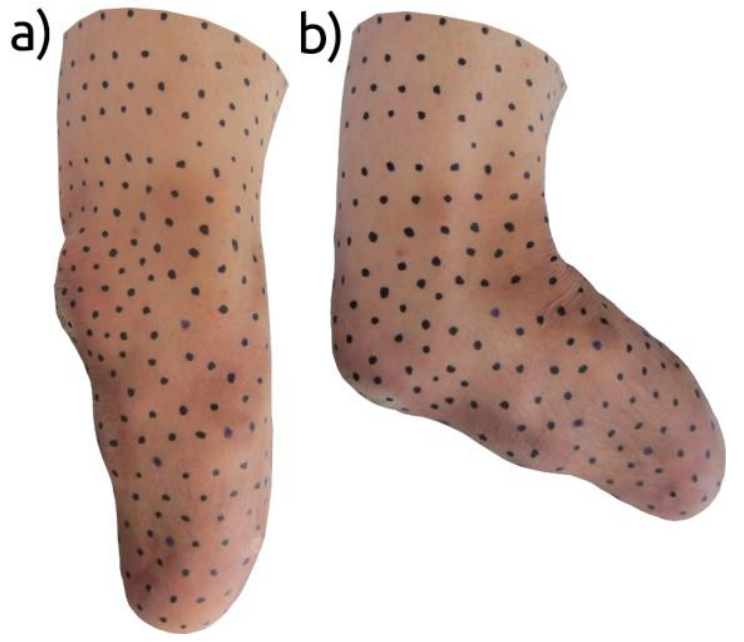

Fig. 2. Visualization of a typical marker pattern on the skin surface in the a) extended and b) flexed knee posture of the same residual limb.

\section{Step (2-3): Image Acquisition, and textured 3D surface model reconstruction}

Once the marker pattern is applied, the research participant is positioned on his stomach with the left residual limb extending over the edge of a raised imaging platform. The imaging platform is draped with a white sheet in order to maintain lighting uniformity during data collection. White balance is manually set against the white sheet at the start of all data collection sessions. This also reduces interference from background objects, movement, and color variations. After the white balance is set, a series of digital photographs (illustrated as arrays in Step 2 of Fig. 1) are acquired $360^{\circ}$ around each residual limb using a standard point and shoot camera in automatic shooting mode without flash (Canon, IXUS 220 HS, shutter speed 1/60 s, aperture f2.7, resolution 1984 x 1488 pixels). The $2 \mathrm{D}$ photos are uploaded into the freeware 123D
Catch (Autodesk, San Rafael, CA) to construct a textured 3D surface model (a 3D model made of vertices, faces, and color information). 123D Catch has cloud-based, desktop, and mobile platform versions designed to interface with cameras ranging from point-and-shoot to cell phone cameras, negating the need for high-end equipment or manual calibration. Given this recreational nature, camera specifications for photo acquisition are very general. In order to optimize the quality of the $3 \mathrm{D}$ model output, it is important to acquire 2D photos based on the 123D Catch guidelines [17]. These guidelines suggest at least $5^{\circ}-10^{\circ}$ overlap between sequential photographs. This was achieved by acquiring 20-40 images for each knee posture, extended and flexed. The optimal distance was determined by maximizing the residual limb in the camera field of view for each frame. This also ensures consistency of model output regardless of patient variability.

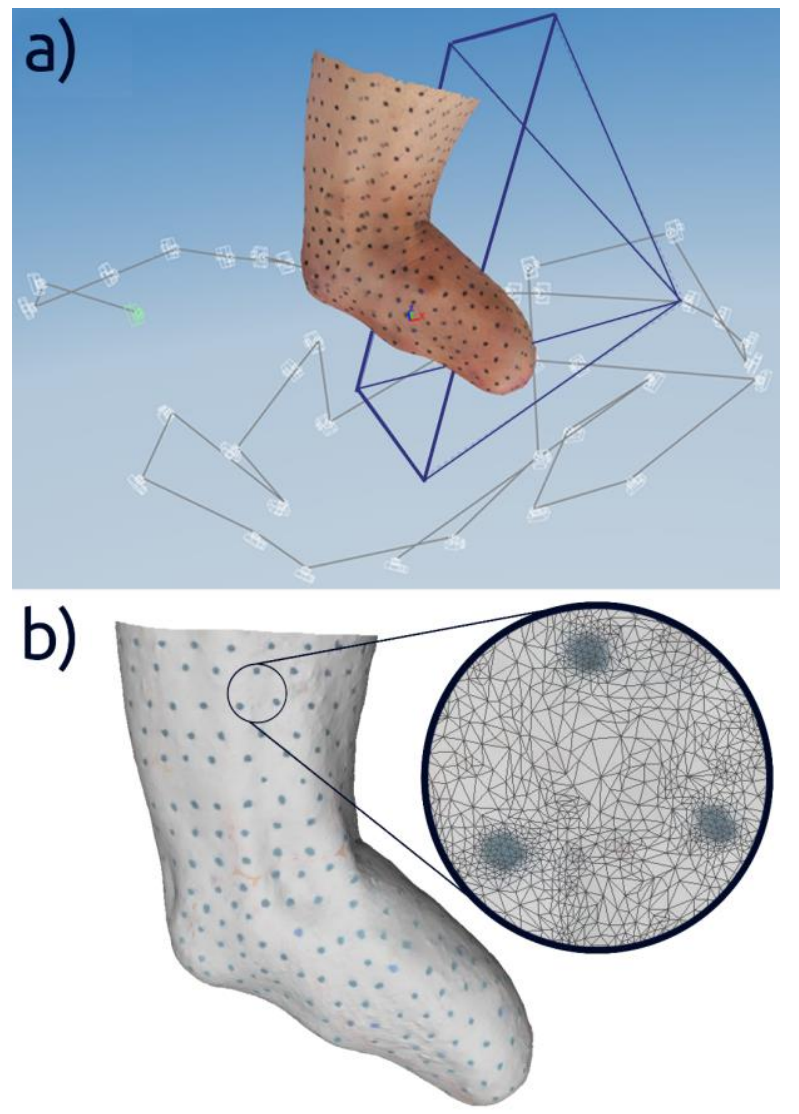

Fig. 3. The $2 \mathrm{D}$ photos are output as a textured $3 \mathrm{D}$ surface model in a) $123 \mathrm{D}$ Catch, illustrating camera orientations (grey path lines) and locations (white camera schematics) with respect to the reconstructed surface, and b) the model is imported into MeshLab, the inset illustrates the refined nature of the mesh at marker locations enabling centroid selection (Step 4).

Image acquisition is completed for both the extended and flexed knee positions of the left residual limb (n1), followed by the extended and flexed positions of the right residual limb (n2), resulting in a total of four independent model renderings. It is important to note that the research participant arbitrarily held his knee in the fully extended and flexed positions when asked to first straighten his knee and then flex his knee. These verbal commands resulted in $\mathrm{n} 1$ having an actual $54^{\circ}$ flexed joint angle, and $\mathrm{n} 2$ having an actual $69^{\circ}$ flexed joint angle. The total 
time required for experimental completion (marker application and imaging) of a single residual limb in both flexed and extended postures is approximately 1 hour.

Fig. 3a illustrates typical orientations and locations of the camera during collection of $2 \mathrm{D}$ images $360^{\circ}$ around the residual limb. 123D Catch automatically corrects for distance and orientation variations during model construction. Each high resolution model reconstruction in 123D Catch takes 1-3 hours, depending on freeware stability. The high resolution textured 3D surface model from 123D Catch is imported into MeshLab freeware, (MeshLab 64-bit, v1.3.4, Institute of Science and Information Technology-National Research Council, Italy). MeshLab renders the model as triangulated surface meshes composed of approximately 100000 vertices and 200000 triangular faces. Fig. $3 \mathrm{~b}$ shows the refined nature of the MeshLab triangulated surface mesh at marker locations. The inset of Fig. 3b shows a close-up of the textured triangulated mesh, highlighting three markers. At marker locations and other areas of non-uniform shape or non-uniform texture, the surfaces become very refined. This ensures that the markers locations are captured by a large number of triangles on the textured surface model.

\section{Step (4) Marker centroid selection and model construction}

The model is manually scaled based on the previously described $2 \mathrm{~cm}$ spacing between two markers in the most proximal row of the residual limb. After scaling the model, marker centroids are manually selected using a manual MeshLab tool. Since the triangulated mesh is most refined at the marker locations shown in Fig. 3b, many mesh vertices are available across each marker. This enables accurate centroid selection of the marker. Fig. 4 shows a typical triangulated surface constructed from the marker centroids. The extended posture is shown on the left, and the flexed posture is shown on the right. The red, green, and blue points are vertices of the same triangle in the extended and flexed posture for point correspondence (Step 5).

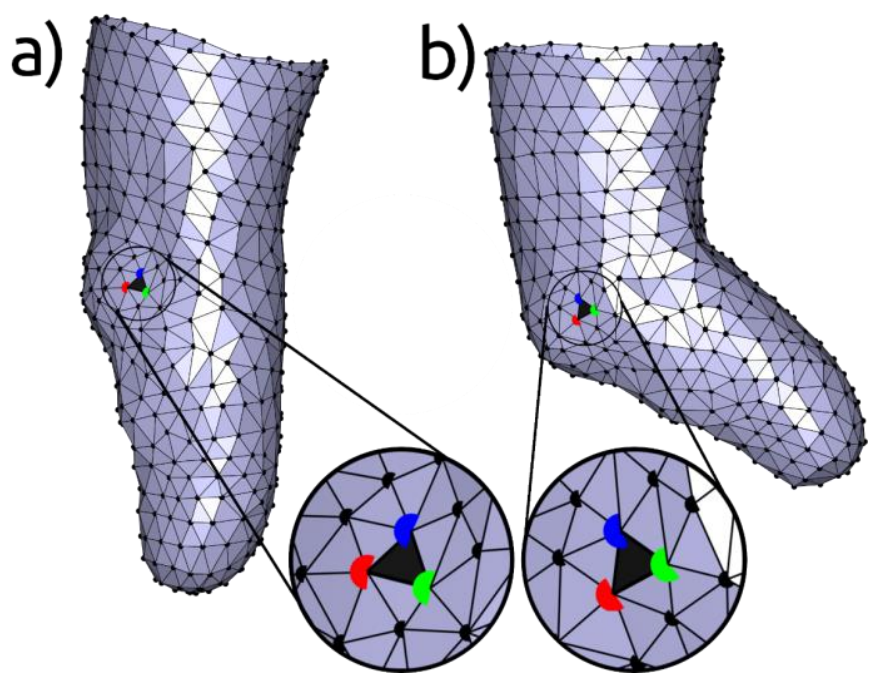

Fig. 4 The triangulated marker surfaces for a limb in the a) extended, and b) flexed postures. The red, green and blue points illustrate vertices of a corresponded triangle, which is shaded black for visualization.
The resulting marker centroid point cloud is exported from MeshLab and imported into MATLAB (R2014b, MathWorks, Natick, MA, USA) for further processing. The manually selected marker centroids create a coarser triangulation whose vertices consist of all the marker centroids. This triangulated surface is called the marker surface model. A publicly available MATLAB script [18], [19] is used to compute a Delaunay triangulation (a triangulation such that for each triangle, the circumcircle only encapsulates the triangle's vertices) of the marker centroid point cloud.

\section{Step (5) Point correspondence}

Since the marker pattern allowed for the creation of a near equilateral triangulated surface (Fig. 4), the shortest diagonal between four adjacent points remains invariant across the expected physiological states of deformation. Consequently, the resulting Delaunay triangulation is also invariant, facilitating unique identification and point tracking across posture changes. As a result, the number of triangles is constant and identifiable between two postures of the same object [20] and vertices can be uniquely corresponded between data sets. Triangles are corresponded using a mesh-growing algorithm [19]. A seed triangle is first manually identified on both postures (as highlighted by red, green, and blue points in Fig. 4). After this manual initiation, auto-correspondence iteratively propagates across the mesh, since the edges of the corresponded triangle can be uniquely matched to adjacent triangles in both extended and flexed deformations at each iteration.

\section{Step (6) Strain and directionality computation}

The local strain at each triangle is computed using custom MATLAB code [21] and publicly available toolboxes. The corresponded triangulated surfaces allow for 3D displacement computation by subtracting the coordinate sets for extended and flexed posture. The displacement at each triangle is then decomposed into translation, rotation, and stretch via an affine transformation [22], [23]. The in-plane maximal and minimal principal stretches $\lambda_{1}$ and $\lambda_{2}$ and principal stretch directions can be obtained using singular value decomposition of the transformation matrix, since the principal stretches and principal stretch directions relate to the singular values and singular vectors. The deformation process of a triangular element is illustrated with a concentric unit circle in Fig. 5a, where $\lambda_{1}=\lambda_{2}$. In Fig. $5 b$, the set of stretches acting along the principal axes have deformed the circle into an ellipse, where $\lambda_{1}>1$ (red) defines a tensile stretch, and $\lambda_{2}<1$ (blue) defines a compressive stretch. The principle strains for each triangle can be computed from the stretches. We calculate true (logarithmic) strain, defined as: $\varepsilon_{i}=\ln \left(\lambda_{i}\right)$, for each triangle to measure large strains that occur during flexion of the knee joint, as opposed to engineering strain, which is not suitable for measuring strain across such large deformations [24]. The mean principal strain is defined as: $\frac{1}{2}\left(\varepsilon_{1}+\varepsilon_{2}\right)$, the maximum shear strain defined as: $\frac{1}{2}\left|\varepsilon_{1}-\varepsilon_{2}\right|$. 
a)

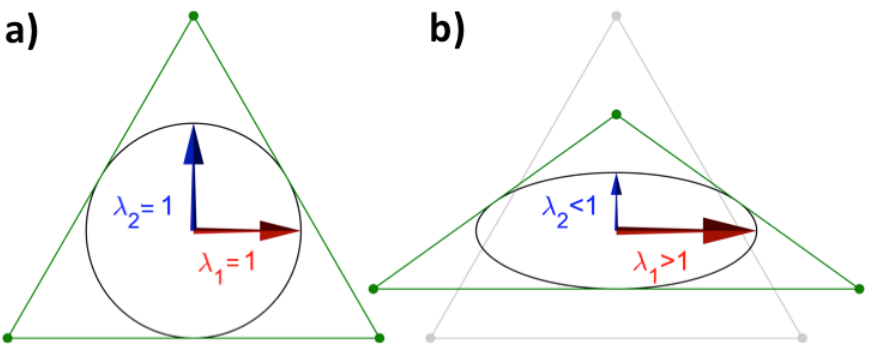

Fig. 5. The deformation process of a reference triangular element is illustrated with a concentric unit circle in a) an (undeformed) circle where $\lambda_{1}=\lambda_{2}$, and b) a (deformed) ellipse, where the maximum $\left(\lambda_{1}>1\right)$ and minimum $\left(\lambda_{2}<1\right)$ principal stretches are shown as red and blue arrows respectively.

The directionality of deformation is also of interest since this may inform the design of custom mechanical interfaces. Of particular interest are the principal strain directions, and the Lines Of Non-Extension (LONE). LONE can be conceptualized as directions where the deformed ellipse in Fig. $5 \mathrm{~b}$ intersects the unit circle in Fig. 5a, this is illustrated in Fig. 6 . It can be seen that for these directions no radial extension or compression is observed at this state of deformation [15]. Depending on the state of deformation either all directions (if $\lambda_{1}=\lambda_{2}=1$, i.e. an undeformed state), a single direction (if only one principal stretch deviates from unity), two directions (i.e. if $\lambda_{1}>1 \wedge \lambda_{2}<1$ such as in Fig. 6), or no direction at all (if both stretches are tensile or if both stretches are compressive i.e. $\left.\left.\left(\lambda_{1}>1 \wedge \lambda_{2}>1\right) \vee\left(\lambda_{1}<1 \wedge \lambda_{2}<1\right)\right)\right)$ can be identified as LONE. It may also occur that stretches are very similar. Therefore LONE are here considered significant only if $\left|\lambda_{1}-\lambda_{2}\right|>0.05$. LONE may be identified at angles $\pm \theta$ with respect to the local first principal strain direction:

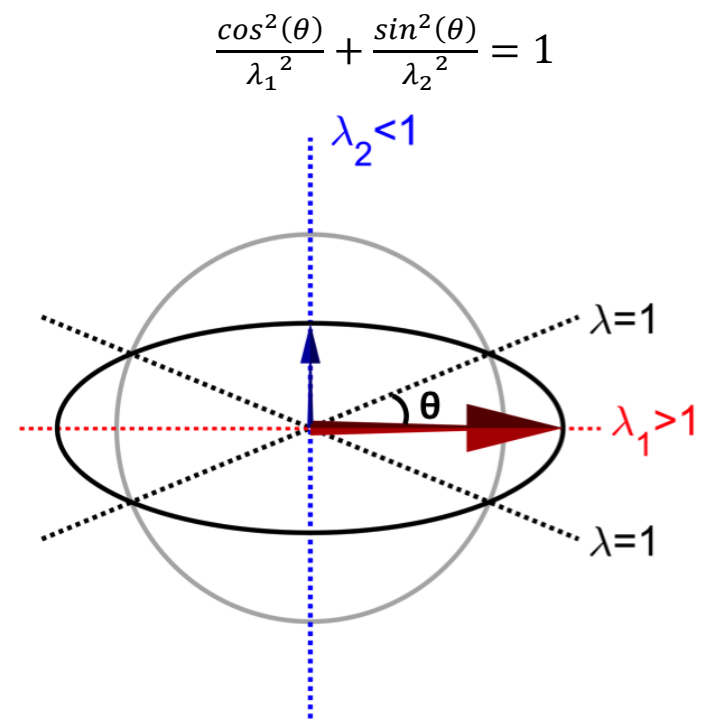

Fig. 6. A unit circle (grey) deformed to an ellipse (black). The circle-to-ellipse deformation has two directions of non-extension located at $\theta= \pm 22.5^{\circ}$ where $\lambda=1$ (LONE), shown using black dotted lines. The maximum $\left(\lambda_{1}>1\right)$ and minimum $\left(\lambda_{2}<1\right)$ principal stretches are shown as red and blue arrows respectively.

It is important to note that LONE may exist in the presence of circumferential strains, strains orthogonal to the triangle, and large displacements, and rotations. For example, the schematic shown in Fig. 6 suggests LONE directions exist at $\pm 22.5^{\circ}$, but these lines started out at $\pm 45^{\circ}$ in the initial state, and have therefore rotated despite the overall deformation lacking a rotational component. It should also be noted that, although LONE directions may be identified for a particular state of deformation, this does not imply that these lines remained a LONE direction throughout the entire history of the deformation.

The local maximum and minimum principal strain, and LONE directions for a flexed posture, are shown for each triangle in Fig. 7 as red, blue, and black line elements respectively. The inset illustrates a single triangle within the surface mesh.

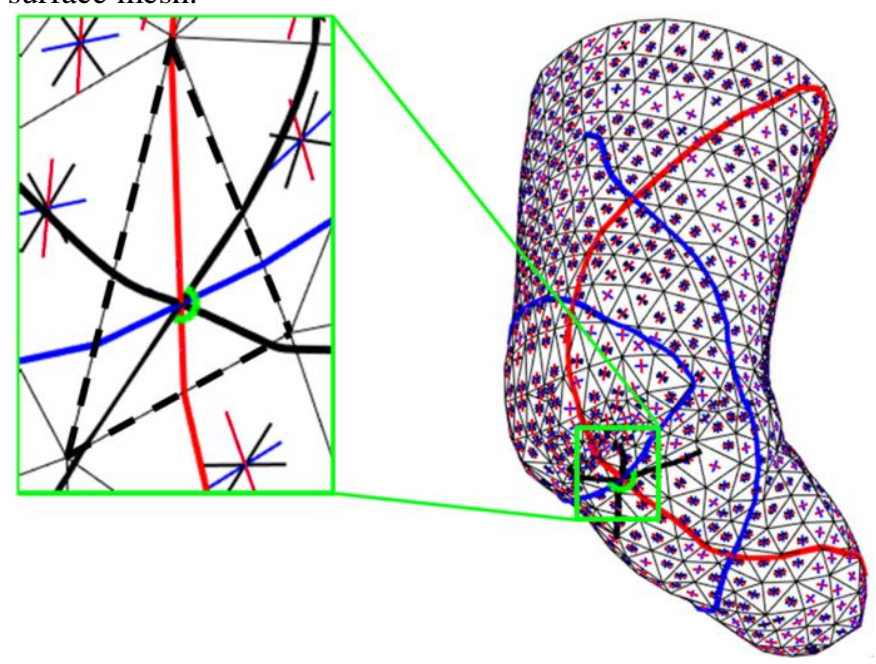

Fig. 7. Colored vectors indicate the local direction of maximum principal strain (red), minimum principal strain (blue), and LONE (black) for each triangle. Reconstructed continuous lines for these measures, for the triangle highlighted by a dashed line in the inset, are shown in red, blue and black respectively.

These multi-linear lines propagate by connecting continuous directional segments across adjacent triangular edges. By definition, the principal strain directions occur once per triangle, are mutually orthogonal, and exist for all triangles. However as mentioned above, zero, one, or two LONE may be identified for a given triangle occurring at varying orientations. If two LONE exist, both directions propagate (see crossing black lines in Fig. 7 inset). If no LONE exists, the line propagation terminates locally.

\section{Evaluation of methodology performance}

Strain data is calculated directly from the obtained transformation matrices defining the deformation between a triangle in extended and flexed states, detailed above in Step (6). The source of error in our results stems from discrepancies between the actual and measured marker locations. These are the focus for evaluation of methodology performance. The marker location error is due to two main effects: 1) 123D Catch model inaccuracies, and 2) manual selection of marker centroids. First, since each model is rendered in arbitrary units using 123D Catch, scaling is required to obtain appropriate dimensions. The scaling error has a global effect due to the manual selection of two known point centroids $\sim 2 \mathrm{~cm}$ apart. Second, the marker centroid error has a local effect, resulting in 
local displacement and strain deviations stemming from two sources; a digital error (e.g. surface shape error) inherent to free-hand acquisition of individual photos, and human error from manual centroid selection of each marker dot (Fig. 2).

In order to evaluate methodology performance and quantify these error sources, a calibration object is 3D printed (Connex 500, Stratasys, Eden Prairie, MN, USA), illustrated in Fig. 8. The calibration object is a rigid body that has a similar size and shape as a residual limb but experiences zero strain. The calibration object is designed with a similar marker pattern and a point spacing $(\sim 2 \mathrm{~cm})$ as defined above in Steps 1 and 2, allowing for near uniform surface triangulation, Steps 3 and 4.

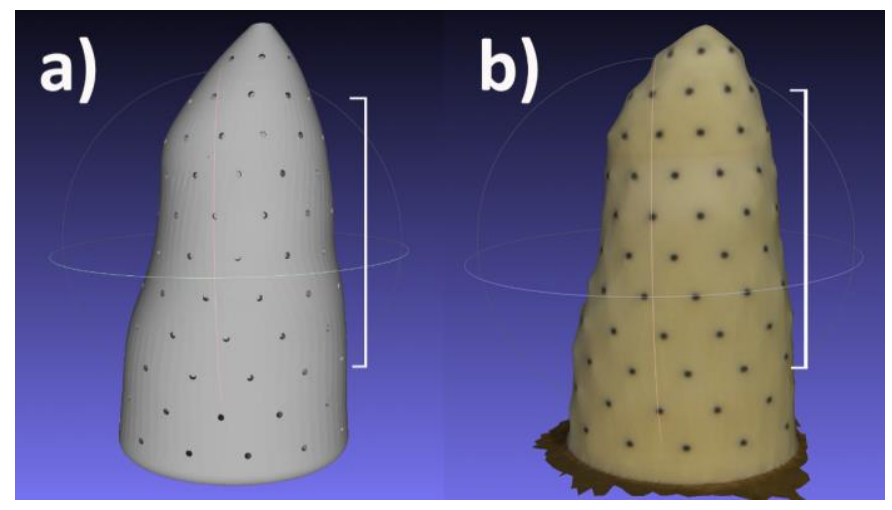

Fig. 8. The calibration object is shown as the a) ground truth digital CAD file, and b) rendered 3D model on a pedestal. Each dark colored point is separated by approximately $2 \mathrm{~cm}$ at known locations. The vertical white bracket indicates the region from which error is computed.

The known marker locations are taken as true coordinates and can be obtained directly from the computer aided design (CAD) file (Fig. 8a). The calibration object is placed stationary on a pedestal to render a 123D Catch derived 3D model (Fig. 8b). The vertical white bracket indicates the region from which error is computed. An open-source MATLAB Toolbox [25][26] is used to perform rigid registration and statistical computation of the $\mathrm{CAD}$ model to the rendered 3D model. Scaling error can be determined from the scaling required to minimize the difference between the rigidly registered surfaces. Once the scaling factor is corrected, difference vectors that define the point location determination error are computed.

\section{RESULTS}

\section{A. Characteristic Skin Strain profiles}

Fig. 9 and Fig. 10 represent histograms of characteristic mean principal and maximum shear strain profiles, respectively. Each bar in the histograms of Fig. 9 and Fig. 10 represents the fraction of the total surface area that experiences magnitudes of mean principal strain and maximum shear strain. The mean principal strain distribution in Fig. 9 shows a larger presence of high strain magnitudes in $\mathrm{n} 2$ compared to $\mathrm{n} 1$. This difference is consistent with $\mathrm{n} 2$ knee flexion being $15^{\circ}$ more acutely flexed at a joint angle of $69^{\circ}$, whereas $\mathrm{n} 1$ had a $54^{\circ}$ flexed angle. In Fig. 10, there is a greater presence of low maximum shear strain (from $0-0.1$ ) in $\mathrm{n} 1$ relative to $\mathrm{n} 2$, and a larger presence of high maximum shear strain (above 0.1 ) in $\mathrm{n} 2$ relative to $\mathrm{n} 1$. As in Fig. 9, this difference is consistent with $\mathrm{n} 2$ knee flexion being $15^{\circ}$ more acute than the $\mathrm{n} 1$ knee flexion. The anatomical mapping of mean principal and maximum shear strain fields, maximum and minimum principal strain direction lines, and directional LONE, for legs $\mathrm{n} 1$ and $\mathrm{n} 2$ are displayed in Fig. 11 and Fig. 12 respectively.

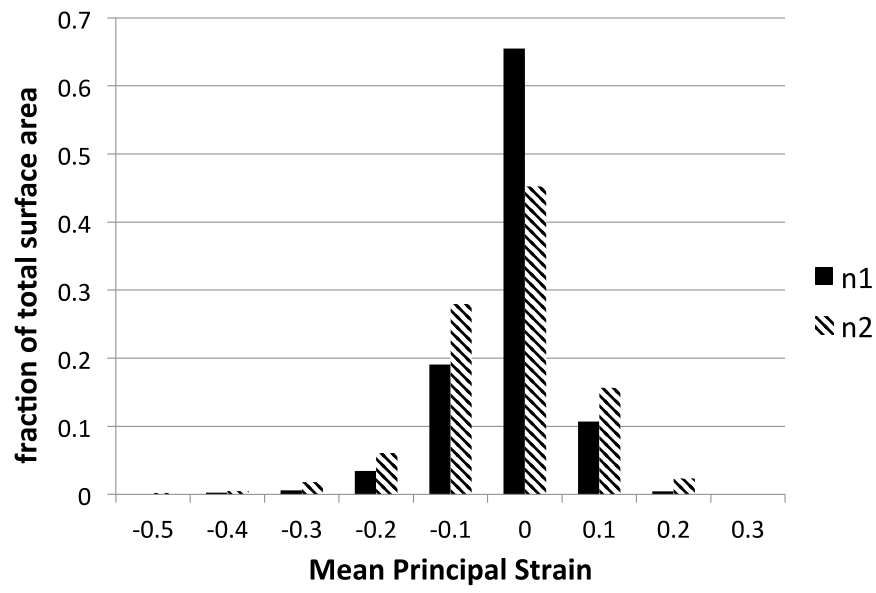

Fig. 9. Mean principal strain histogram distribution. The fraction of the total surface area that experiences magnitudes of mean principal strain for residual limbs $\mathrm{n} 1$ and $\mathrm{n} 2$.

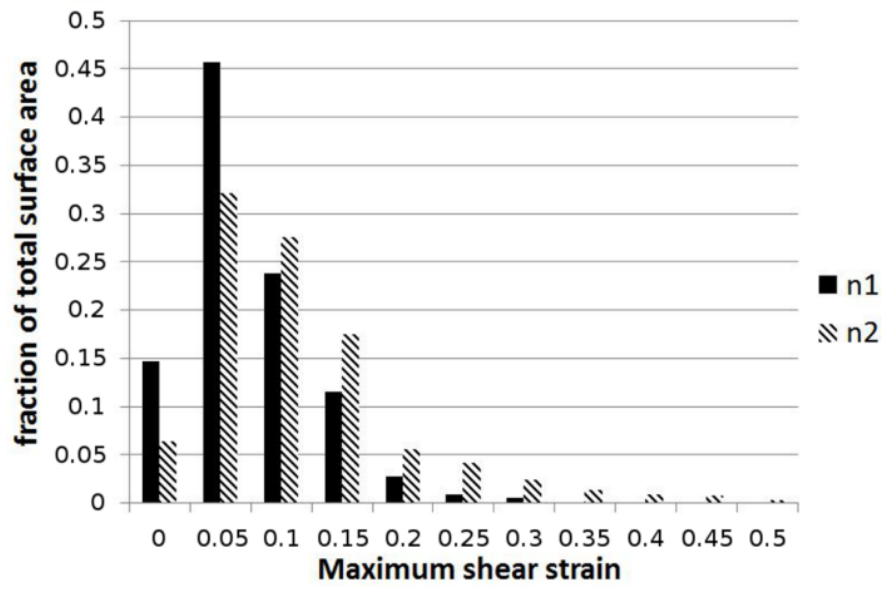

Fig. 10. Maximum shear strain histogram distribution. The fraction of the total surface area that experiences magnitudes of shear strain for residual limbs $\mathrm{n} 1$ and $\mathrm{n} 2$.

\section{B. Mean principal strain}

Mean principal strain data for $\mathrm{n} 1$ and $\mathrm{n} 2$ are shown in Fig. 11(a-d) and Fig. 12(a-d) respectively. Peak tensile mean principal strains $(\sim 0.3)$ are observed on the anterior surface of the knee in the proximal region of the patella, while peak compressive mean principal strains $(\sim-0.5)$, are observed on the posterior surface of the knee.

\section{Maximum shear strain}

The maximum shear strain for $\mathrm{n} 1$ and $\mathrm{n} 2$ are shown in Fig. 11(e-h), and Fig. 12(e-h) respectively. These show the peak maximum shear strain to be $\sim 0.3$, which is found on the posterior surface of the knee. The maximum shear strain profile was qualitatively similar between $\mathrm{n} 1$ and $\mathrm{n} 2$, although $\mathrm{n} 2$ displayed larger deformations compared to $\mathrm{n} 1$ simply because the $\mathrm{n} 2$ knee flexion angle was $15^{\circ}$ more acute than the n1 knee angle. 
a)

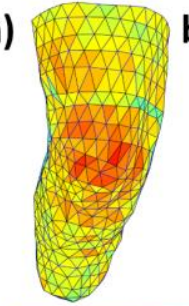

b)

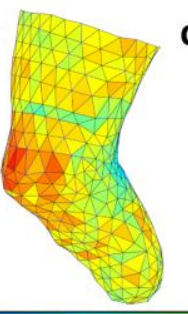

c)

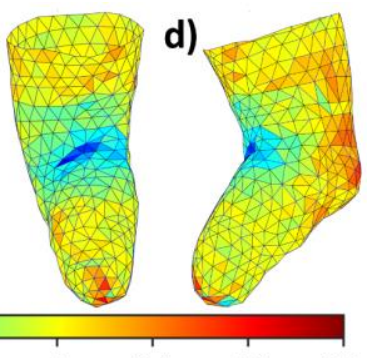

$\begin{array}{ccc}-0.5 & -0.4 & -0.3\end{array}$

e)

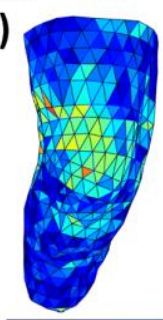

$\begin{array}{lll}-0.3 & -0.2 & -0.1\end{array}$

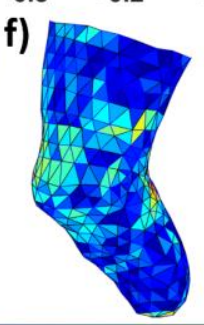

0.1
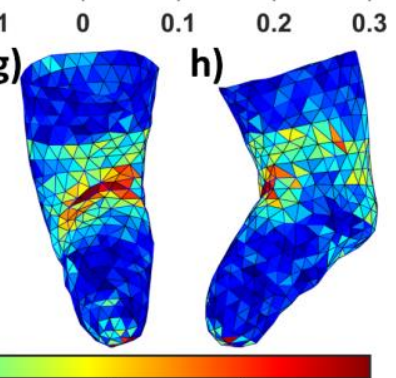

h)

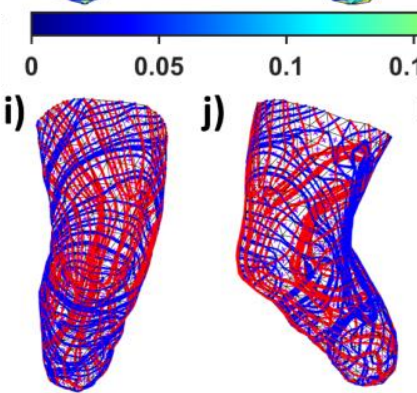

k)

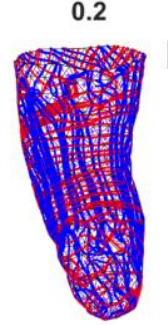

m)

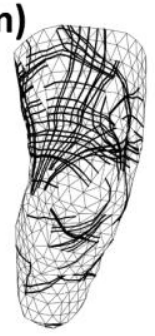

n)

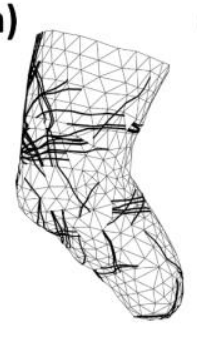

o)

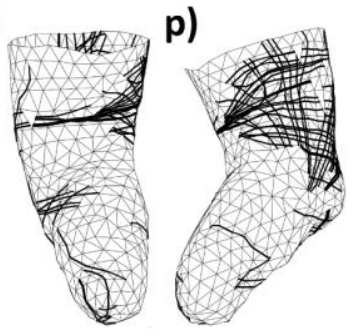

Fig. 11. Skin strain mapping of leg $\mathrm{n} 1$ viewed from the knee anterior, lateral, posterior, and medial surfaces for: (a-d) mean principal strain, (e-h) maximum shear strain, (i-l) maximum (red) and minimum (blue) principal strain lines, and (m-p) connected LONE (black).

\section{Principal strain lines}

The maximum principal strain direction line data for $\mathrm{n} 1$ and $\mathrm{n} 2$ are shown in Fig. 11(i-l) and Fig. 12(i-1), respectively. These lines (red) pass longitudinally across the anterior surface of the knee, and circumferentially across the posterior surface of the knee. Conversely, lines of minimum principal strain (blue) are by definition orthogonal to the lines of maximum principal strain, and thus pass circumferentially across the anterior surface of the knee, and longitudinally across the posterior surface of the knee.

\section{E. Lines OfNon-Extension (LONE)}

Directional LONE for $\mathrm{n} 1$ and $\mathrm{n} 2$ are illustrated as discontinuous black lines in Fig. 11(m-p) and Fig. 12(m-p) respectively. LONE propagation terminates if no LONE is locally identified, such as regions across the patella, but are observed to be more densely located in regions of low strain. Since the existence and multitude of LONE depend on the state of deformation, it is natural that they may not be continuous.

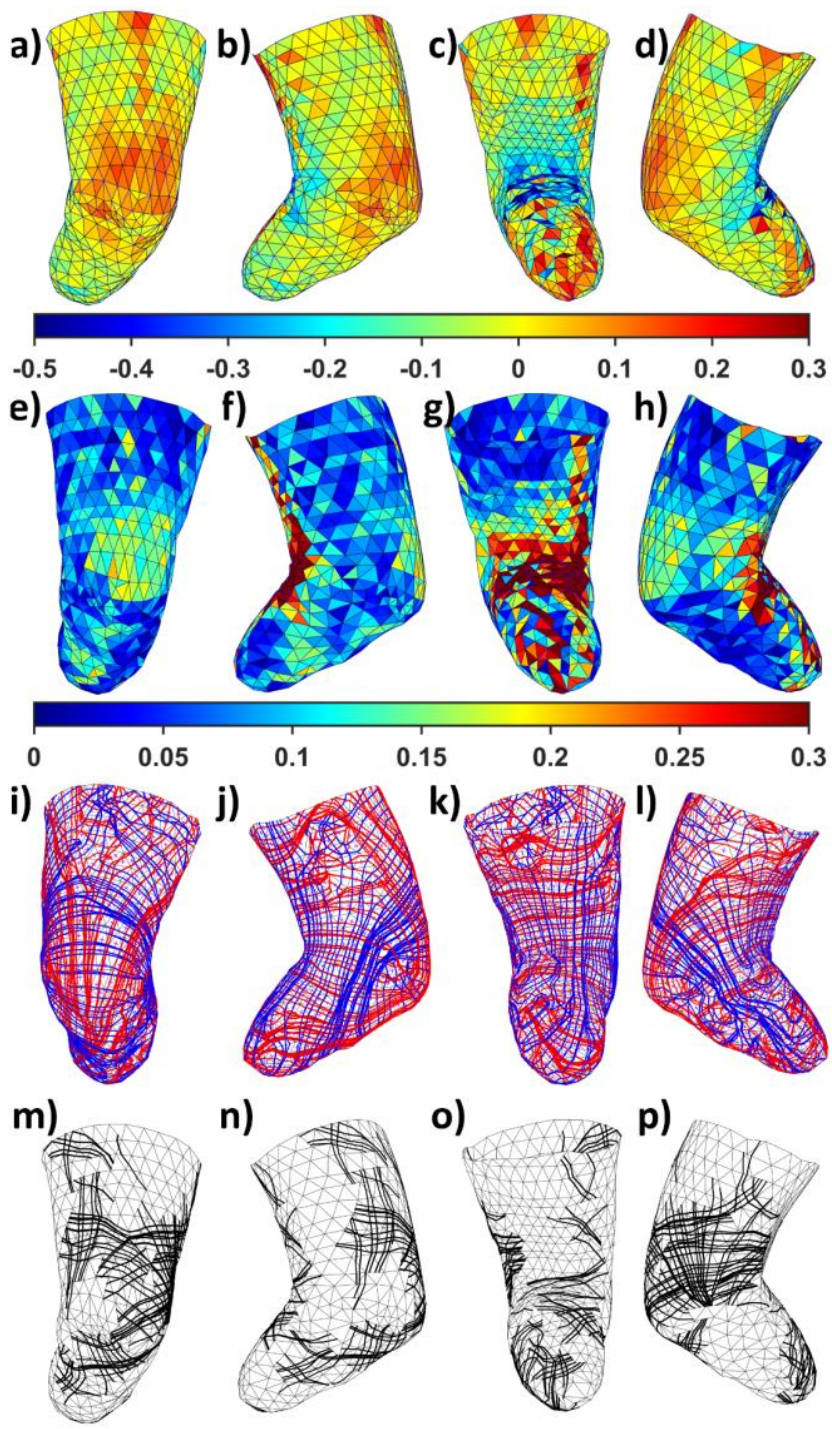

Fig. 12. Skin strain mapping of leg $\mathrm{n} 2$ viewed from the knee anterior, lateral, posterior, and medial surfaces for: (a-d) mean principal strain, (e-h) maximum shear strain, (i-1) maximum (red) and minimum (blue) principal strain lines, and (m-p) connected LONE (black).

\section{F. Evaluation of methodology performance}

The scaling error of the calibration object is computed to be 0.93 , indicating manual scaling success within $7 \%$. The scaling error is visible in Fig. 13a, where the ground truth CAD file is shown in blue, and the $7 \%$ dilated model is overlaid in red. Point location determination error is visualized in Fig. 13b as points colored towards the magnitudes of difference vectors, which are the distances $(\mathrm{cm})$ between points on the CAD surface and the model surface after $7 \%$ corrective scaling. The point location difference vector data presents with an overall standard deviation of $0.05 \mathrm{~cm}$. The maximum point location distance was $0.16 \mathrm{~cm}$, visible as a red colored vertex in the lower left quadrant of Fig. 13b. The mean point location distance is 0.08 $\mathrm{cm}$. 


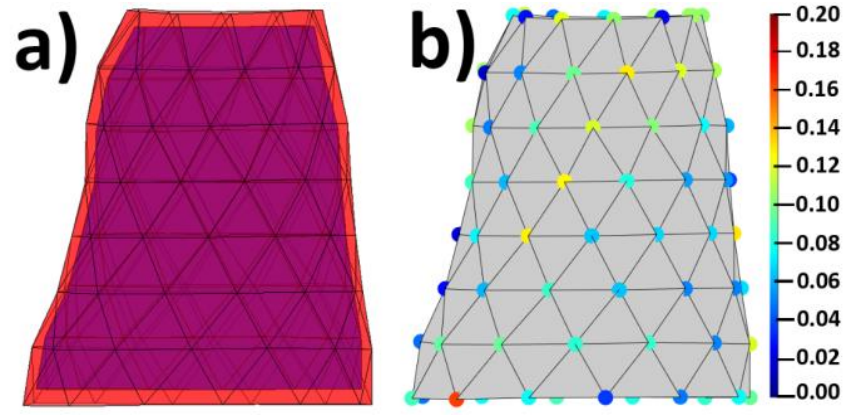

Fig. 13. Methodology performance visualized as a) a 7\% scaling error dilation given the digital CAD file (blue) overlaid with the rendered model (red), and b) point location determination error shown as points colored towards the distance $(\mathrm{cm})$ between the points for the CAD file and the rendered model.

\section{DISCUSSION}

The measurement of skin strain resulting from natural movement of a biological joint derives importance from a myriad of applications such as apparel, shoes, and wearable robotics. An inexpensive approach to skin strain measurement unconfined to a lab is paramount to applications that necessitate mobile yet accurate estimates. Examples include persons in the field where expensive and bulky equipment may not be available. In this study, we describe a mobile approach to quantify whole-limb skin strain across large joint angles using a standard point-and-shoot digital camera and freeware to analyze 3D quasi-static deformations. We apply and evaluate this skin strain methodology for two transtibial residual limbs in a flexed knee posture, as such data may inform the design of future prosthetic socket interfaces.

Macro-scale strain patterns Fig. 11 and Fig. 12 are clearly visible using this method and may benefit clinical needs where global patterns of skin strain are important. As expected for the knee flexion exercise investigated, the anterior patella region of both left and right residual limbs exhibited predominantly tensile strains (a peak mean principal strain of $\sim 0.3$ was observed), while the posterior patella region exhibited predominantly compressive strains (a peak mean minimum principal strain of $\sim-0.5$ was observed). The latter region also presented with the peak maximum shear strains $(\sim 0.3)$, consistent with the fact that this skin region undergoes the most shape change as the knee is flexed. The skin around the quadriceps and hamstrings (proximal regions), and the gastrocnemius and soleus (distal regions) experience relatively low strains as these regions presented with near-zero mean principal strains and low maximum shear strains.

Fig. 11(i-l) and Fig. 12(i-l) show directional principal strain data for legs $n 1$ and $n 2$, respectively. The anterior surface of the residual limb displays longitudinal mappings (coronal plane) that are predominantly due to the maximum principal strain (red), coupled with circumferential mappings (transverse plane) of minimum principal strain (blue). These directional strain fields reverse orientation on the posterior surface of the residual limb, displaying longitudinal mappings (coronal plane) that are predominantly minimum principal strain (blue), coupled with the circumferential mappings (transverse plane) of maximum principal strain (red). These patterns are consistent with what is expected for the knee flexion exercise, i.e. that anterior and posterior present opposing behavior during flexion. The anterior side is dominated by tensile strains while the posterior side is dominated by compressive strains.

Mapped LONE are illustrated in Fig. 11(m-p) and Fig. 12(mp) as black lines that indicate local directions where extension or compression are not present during joint flexion. LONE presented herein follow similar characteristic profiles as the first observations of LONE [15]. However, while literature LONE are illustrated as continuous lines throughout entire sections of the body, our results are discontinuous across the residual limb. The discontinuities are expected based on the definition of the LONE phenomenon described in Section II(B), Step(6), and have been previously observed [2]. In the presented work, LONE was computed locally if $\left|\lambda_{1}-\lambda_{2}\right|>$ 0.05 . Future investigations to improve the continuity of LONE derived propagated lines could optimize both the threshold and the line propagation algorithm for inclusion of nearlyundeformed regions. Although the LONE phenomenon has garnered promise for mechanical interface design, other directional strain data may prove more useful in the future. For example, lines of minimum absolute strain may be a candidate for future research, since these are continuous and coincide with LONE or principal strain directions. Further research is required to evaluate the utility of directional strain data. Strain directions are subject to time varying rotations, while LONE are shown to also be transient.

The total error associated with the presented methodology is evaluated using a ground truth rigid calibration object. Two main modes of error are distinguished; a global scaling error, and a local point location determination error. The former is evident in Fig. 13a as a visible 7\% dilation of the rendered model overlaid with the CAD model. This global error can be addressed in future trials by including a rigid body of known dimensions in each frame to serve as a stationary scaling reference (e.g. a rigid sphere of known size). The latter is illustrated in Fig. 13b as points (vertices) colored towards the magnitudes of each difference vector. The mean point location distance between the CAD ground truth and the rendered model is $0.08 \mathrm{~cm}$, the overall standard deviation for point location difference vectors is $0.05 \mathrm{~cm}$, and the largest distance is 0.16 $\mathrm{cm}$. Point location errors can be improved by using automatic point detection.

Developing a multi-camera imaging rig to enable simultaneous sub-second image acquisition would provide a static platform from which to ensure a constant focal length for improved model reconstruction, helping to address limitations of the uncontrolled variables of the freeware. Although 123D Catch is a useful tool, the input requires 20 to 40 still-frame photos. The 10-15 minutes necessary to acquire such a large number of sequential images causes fatigue in human research participants, who are asked to remain motionless for the duration of data acquisition. Developing the above mentioned imaging system may help prevent fatigue and reduce error from motion artifacts inherent to in vivo imaging of the residual limb. Furthermore, video capture could be implemented using the above described imaging rig. Video capture would enable dynamic skin strain analysis while retaining still-frame data. 
Future work also includes investigation of optimal computation times relative to triangulation methods and data analysis, using reality capture software that has improved capabilities with the resolution of a $35 \mathrm{~mm}$ camera (ReCap, Autodesk, San Rafael, CA). The stationary scaling reference discussed above to correct for global error could also be useful for minimizing motion artifacts.

Comparison of strain findings to the literature is challenging since methodologies, deformation regimes, and analysis methods may differ significantly. In addition, results may differ between research participants, and no gold standard yet exists. Our strain results are, however, within a similar range of values reported in an earlier motion capture study [2], which discussed a need for marker position error analysis and marker density refinement. Our study presents both a refinement in marker density quantified by marker positon error analysis. Further, a significant cost reduction is achieved using the low-cost methodology detailed here, compared to motion capture or DIC based strain imaging. DIC has also been used to investigate local skin strain [9]. Although DIC offers superior resolution, applications have been limited to measurements near a loading site and approximately planar geometries. The work by Manthey et al. 1995 [27] focused on surface strain in a sheet metal object, based on the application of a regular grid pattern. Such an approach is feasible for objects with initially planer or highly regular geometries. To cope with the complex geometry of the human body, we employ a marker pattern instead. This makes the experimental design very flexible as few constraints are placed on the mesh configuration, which is unknown a priori and may significantly vary between subjects. Given the elaborate installations required for motion capture and DIC based skin strain analysis, we present a low cost, highly accessible, and mobile alternative.

As demonstrated here, measurement of skin strain during an exercise, such as joint bending, can be post-processed to provide directional strain information. The directional data include lines of non-, minimum, and maximum extension. In the absence of a garment or wearable device, the measurement provides the skin's natural deformation occurring during exercise. To optimize the comfort of a garment or wearable device, its properties at the interface with the skin can be modified to accommodate the natural skin deformation pattern. For example, an anisotropic biomechanical interface can be relatively compliant by design (in terms of resisting tension or compression) in the direction of maximum strain, thereby reducing relative motion and shear forces between the skin and the device. Conversely, the interface material can be reinforced along the lines of non-extension or minimum strain to enable maximum motility of the skin while providing targeted support. As an example, following the work by Iberall et al. [15], [28], researchers have proposed the use of the lines of non-extension in the design of space suits [29][30][31] e.g. incorporating local directional adjustment of fabric reinforcement and contractile properties in parallel to natural skin deformation. Similarly, in light of the potential application of the presented work, strain directionality for transtibial amputee lower limb joint bending may be used to locally enhance or reduce the stiffness of the prosthetic liner or socket to limit relative motion and shear forces at the skin surface. The aim of such an anisotropic interface may be to minimize alteration of the natural deformation pattern. It should be noted that we have considered only the case of strain on an unloaded residual limb. In reality, the presence of wearable technology such as a prosthetic device, will alter skin strain directionality and magnitudes. Weight bearing activity with the use of a mechanical interface will introduce new variables and alter mechanical conditions, dynamically shifting strain fields. This further highlights the importance of the application of the presented techniques in the presence of a prosthetic liner-socket interface during ambulation. In order to assess skin deformation in the presence of the biomechanical interface using the presented methodology, transparent materials for prosthetic devices could be employed. Validated computational biomechanical models [32] (e.g. trained to match experimental strain conditions) may also be used to simulate the deformations occurring at the device-skin interface [33], thereby removing the need for transparent materials. These models can also be combined with iterative adjustment of the interface properties (shape and local anisotropy) to further optimize skin loading and deformation [33]. Future work will investigate the use of the presented strain assessment techniques, coupled with biomechanical modeling, to inform dynamic properties of a patient-specific prosthetic interface (liner and socket).

\section{CONCLUSION}

Understanding skin biomechanics may prove critical to the design of wearable technologies that directly interface with human skin. Rather than relying on bulk production designed with generic characteristics, a uniform process to quantify strain fields using portable and cost effective resources could help advance the development of custom devices by providing quantitative data. These data could potentially be used for the design and fabrication of anisotropic prosthetic liners. For example, lines of maximum strain could dictate where the liner needs to be more compliant, while a less compliant material could be embedded along the lines of minimum strain. Providing a positional error will also be informative of the precision range necessary when considering custom liner design and fabrication. The human body comprises continuously-varying tissue impedances given its multi-tissue composition, and thus the design and manufacture of innovative technology must also reflect this biomechanical variability. It is our hope that the low-cost, skin-strain measurement methodology presented here may inform human-machine interface design with applications in wearable body exoskeletons, prosthetic and orthotic devices.

\section{ACKNOWLEDGMENTS}

The authors thank Luke Mooney for his insightful feedback, as well as Nalini Singh, Andrew Mullen, Rhyse Bendell, and Pierce Hayward for technical assistance. 


\section{REFERENCES}

[1]

H. M. Herr, A. Marecki, and D. M. Sengeh, "Variable Impedance Mechanical Interface," US20130282141 A1, 2013.

[2] A. M. Wessendorf Newman, D.J., A. M. Wessendorf, and D. J. Newman, "Dynamic understanding of human-skin movement and strain-field analysis," IEEE Trans. Biomed. Eng., vol. 59, no. 12, pp. 3432-3438, 2012.

C. W. Oomens, D. H. van Campen, and H. J. Grootenboer, "A mixture approach to the mechanics of skin.," J. Biomech., vol. 20, pp. 877-885, 1987.

[4] R. Groves, S. Coulman, J. C. Birchall, and S. L. Evans, "Quantifying the mechanical properties of human skin to optimise future microneedle device design," Comput. Methods Biomech. Biomed. Engin., vol. 15, no. 1, pp. 73-82, Jan. 2012.

C. Flynn, A. Taberner, and P. Nielsen, "Modeling the mechanical response of in vivo human skin under a rich set of deformations," Ann. Biomed. Eng., vol. 39, no. 7, pp. 1935-1946, Jul. 2011.

[6] L. K. Smalls, R. Randall Wickett, and M. O. Visscher, "Effect of dermal thickness, tissue composition, and body site on skin biomechanical properties," Ski. Res. Technol., vol. 12, no. 1, pp. 4349, 2006.

[7] J. W. Y. Jor, M. D. Parker, A. J. Taberner, M. P. Nash, and P. M. F. Nielsen, "Computational and experimental characterization of skin mechanics: Identifying current challenges and future directions," Wiley Interdiscip. Rev. Syst. Biol. Med., vol. 5, no. 5, pp. 539-556, 2013.

[8] K. M. Moerman, C. a. Holt, S. L. Evans, and C. K. Simms, "Digital image correlation and finite element modelling as a method to determine mechanical properties of human soft tissue in vivo," $J$. Biomech., vol. 42, no. 8, pp. 1150-1153, 2009.

[9] S. L. Evans and C. a Holt, "Measuring the mechanical properties of human skin in vivo using digital image correlation and finite element modelling," J. Strain Anal. Eng. Des., vol. 44, no. 5, pp. 337-345, Jul. 2009.

[10] B. Pierrat, C. Millot, J. Molimard, L. Navarro, P. Calmels, P. Edouard, and S. Avril, "Characterisation of Knee Brace Migration and Associated Skin Deformation During Flexion by Full-Field Measurements," Exp. Mech., vol. 55, no. 2, pp. 349-360, Oct. 2014.

[11] K. A. Bethke, "The second skin approach: Skin strain field analysis and mechanical counter pressure prototyping for advanced spacesuit design," Massachusetts Institute of Technology, 2005.

[12] A. R. Domingues Marreiros, S.P., Martins, J.M., Silva, M.T., Newman, D.J., "Skin Strain Field Analysis of the Human Ankle Joint," in 4th Congresso Nacional de Biomechanica, 2010.

[13] E. W. Obropta and D. J. Newman, "Skin strain fields at the shoulder joint for mechanical counter pressure space suit development," in 2016 IEEE Aerospace Conference, 2016, pp. 1-9.

[14] E. W. Obropta and D. J. Newman, "A comparison of human skin strain fields of the elbow joint for mechanical counter pressure space suit development," in 2015 IEEE Aerospace Conference, 2015, pp. 1-9.

[15] A. S. Iberall, "The Experimental Design of a Mobile Pressure Suit," J. Basic Eng., vol. 92, no. 2, p. 251, Jun. 1970.

[16] M. Nolan, "Limits of two-point discrimination ability in the lower limb in young adult men and women," Phys. Ther., 1983.

[17] Autodesk, "Learn how to use 123d Catch." .

[18] L. Giaccari, "MyCrustOpen.m.”.

[19] L. Di Angelo, P. Di Stefano, and L. Giaccari, "A new mesh-growing algorithm for fast surface reconstruction," CAD Comput. Aided Des., vol. 43, no. 6, pp. 639-650, 2011.

[20] D. T. Lee and B. J. Schachter, "Two algorithms for constructing a Delaunay triangulation," Int. J. Comput. Inf. Sci., vol. 9, no. 3, pp. 219-242, Jun. 1980.
[21] A. T. Marecki, "Skin strain analysis software for the study of human skin deformation," Massachusetts Institute of Technology, 2012.

[22] A. S. Mian, "Representations and Matching Techniques for 3D Free-form Object and Face Recognition," University of Western Australia, 2006.

[23] A. S. Mian, M. Bennamoun, and R. Owens, "Automated 3D modelbased free-form object recognition," Sens. Rev., vol. 24, no. 2, pp. 206-215, Jun. 2004.

[24] G. S. Archer, R.Rl, Cook, N.H., Crandall, S.H., Dahl, N.C., Lardner, T.J., McClintock, F.A., Rabinowitcz, E., Reichenbach, An Introduction to the Mechanics of Solids, 2nd ed. McGraw-Hill Inc., 1972.

[25] K. M. Moerman, A. J. Nederveen, and C. K. Simms, "Image Based Model Construction, Boundary Condition Specification and Inverse Fea Control : a Basic Matlab Toolkit for Febio," Proc. 11th Int. Symp. Comput. Methods Biomech. Biomed. Eng., pp. 7-8, 2013.

[26] K. M. Moerman, "GIBBON (Hylobates Agilis)." 13-Oct-2014.

[27] D. W. Manthey and D. Lee, "Recent developments in a vision-based surface strain measurement system," JOM, vol. 47, no. 7, pp. 46-49, Jul. 1995.

[28] A. S. Iberall, "The Use of Lines of Nonextension to Improve Mobility in Full-Pressure Suits," AMRL-TR. Aerosp. Med. Res. Lab., pp. 1-35, Nov. 1964.

[29] B. Holschuh, E. Obropta, L. Buechley, and D. Newman, "Materials and Textile Architecture Analyses for Mechanical Counter-Pressure Space Suits using Active Materials," in AIAA SPACE 2012 Conference \& Exposition, 2012.

[30] D. C. L. Judnick, "Modeling and testing of a mechanical counterpressure BioSuit system," 2007.

[31] L. Sim, K. Bethke, N. Jordan, C. Dube, J. Hoffman, C. Brensinger, G. Trotti, and D. Newman, "Implementation and Testing of a Mechanical Counterpressure Bio-Suit System,” 2005.

[32] D. M. Sengeh, K. M. Moerman, A. Petron, and H. M. Herr, "Multimaterial 3-D viscoelastic model of a transtibial residuum from invivo indentation and MRI data," J. Mech. Behav. Biomed. Mater., vol. 59, pp. 379-392, Feb. 2016.

[33] K. M. Moerman, H. M. Herr, and D. M. Sengeh, "Automated and Data-driven Computational Design of Patient-Specific Biomechanical Interfaces," Open Sci. Framew., 2016. 


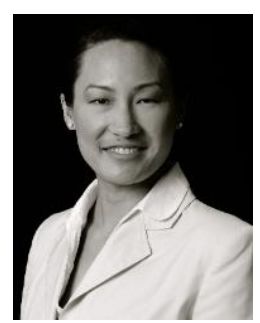

Bevin Lin received a B.S. degree in chemistry (2002) from the University of California (UC), Santa Barbara. She conducted research with the New Technologies Engineering Division at Lawrence Livermore National Laboratory (2003) before completing a Ph.D. in biomedical engineering (2010) from UC Davis. After postdoctoral appointments at the National University of Singapore (2011) and the Massachusetts Institute of Technology (MIT) (2012), Dr. Lin joined the MIT Media Lab in 2013 as a research scientist and lab manager of the Biomechatronics Group and Center for Extreme Bionics. Her research interests include diagnostic imaging, biophotonics, and the human-machine interface.

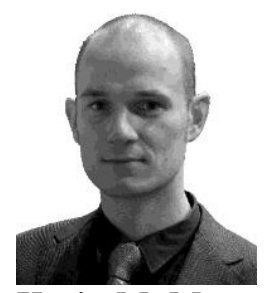

Kevin M. Moerman received a B.Eng. degree in mechanical engineering (2004) from The Hague University of Applied Sciences, The Hague, The Netherlands. He also received a M.Sc. degree (2005) and a Ph.D. degree (2012) in Bioengineering from the University of Dublin, Trinity College, Dublin, Ireland. He was a postdoctoral researcher at the Academic Medical Centre Amsterdam (2011-2015). He is currently a postdoctoral associate at the Biomechatronics Group and Center for Extreme Bionics of the Massachusetts Institute of Technology Media Lab. He is also visiting research fellow at the Centre for Bioengineering, University of Dublin. His main research interests are biomedical device design, soft tissue biomechanics, image-based modeling, and inverse finite element analysis.

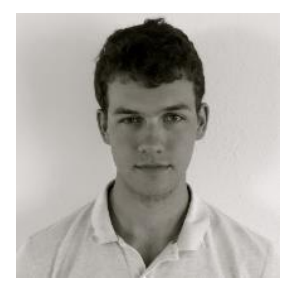

Connor G. McMahan received a B.S. degree (2016) in mechanical engineering from the Massachusetts Institute of Technology (MIT). In 2013, he was a Research Assistant at the University of Colorado, Boulder, where he investigated the fabrication and characterization of gold nanowire meshes. His research interests include prosthetic devices and the biomechanics of movement.

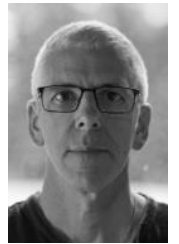

Kenneth A. Pasch received a B.S. (1981), M.S. (1984), and $\mathrm{Ph} . \mathrm{D}$. (1988) degree in mechanical engineering from the Massachusetts Institute of Technology, (MIT) Cambridge, MA. $\mathrm{He}$ is currently a research scientist within the MIT Center for Extreme Bionics, and is a licensed professional mechanical engineer. He has taught courses in mechanical engineering at MIT, the University of Illinois in Chicago, and Tufts University in Boston. Dr. Pasch is named as an inventor on fourteen U.S. patents, and has research interests in mechanical design, control systems, and optimization.

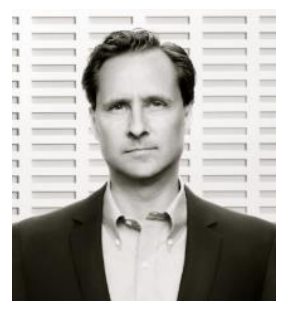

Hugh M. Herr received a B.A. degree in Physics (1990) from Millersville University of Pennsylvania, a M.S. degree in mechanical engineering (1993) from the Massachusetts Institute of Technology (MIT), and a Ph.D. degree in biophysics (1998) from Harvard University. He is Associate Professor within MIT's Program of Media Arts and Sciences, and co-directs MIT's Center for Extreme Bionics. His primary research objective is to apply principles of limb neuromechanics to the design of wearable robotics for human augmentation and rehabilitation. In the area of human augmentation, Professor Herr has built elastic shoes and powered leg exoskeletons for walking and running that decrease metabolic demand. In the area of assistive technology, Professor Herr has developed powered orthotic and prosthetic mechanisms for use as assistive interventions in the treatment of leg disabilities caused by amputation, stroke, cerebral palsy and multiple sclerosis. Herr is the author and co-author of over 150 peer-reviewed manuscripts and patents within the emerging field of biomechatronics. 